\title{
EFFECTS OF CHIA SEED SUPPLEMENTATION ON BIOCHEMICAL MARKERS OF CARDIOMETABOLIC DISEASES IN SPONTANEOUSLY HYPERTENSIVE RATS
}

\author{
L. Costantini*, R. Molinari, and N. Merendino \\ Department of Ecological and Biological Sciences (DEB), Tuscia University, Largo dell’Università snc, 01100, \\ Viterbo. Italy
}

(Received: 3 April 2019; accepted: 15 May 2019)

\begin{abstract}
Some studies suggested a positive effect against cardiometabolic diseases of supplementation of alpha-linolenic acid (ALA, C18:3n-3) rich foods in pathological subjects, even if the total literature is controversial. In order to clarify ALA-rich chia seed action in hypertensive model with the overt pathology and without drug interference, in the present study the biochemical markers of cardiometabolic diseases (endothelin-1, ET-1; nitric oxide, NO; and bradykinin, BK) in Spontaneously Hypertensive Rats (SHRs) were analysed after 5\% chia seed dietary supplementation for five weeks, and compared with the staple raw material wheat and corn. At the end of the experimental period, also plasma antioxidant capacity and inflammatory condition were evaluated. Our results showed that the chia seed group was more oxidized. On the other hand, ET-1 significantly decreased in chia seed group, and there was no difference between groups for NO, BK, and the inflammatory C-reactive protein (CRP). In conclusion, some positive effects of chia seed consumption on cardiometabolic markers in SHRs were observed, despite this the association of chia seeds with antioxidants is suggested to avoid plasma oxidation increase.
\end{abstract}

Keywords: chia seeds, alpha-linolenic acid, endothelin-1, nitric oxide, bradykinin

In their last (2018) report, the American Heart Association declared that there were 3.47 billion adults worldwide with systolic blood pressure of 110 to $115 \mathrm{mmHg}$ or higher in 2015, and 874 million adults had systolic blood pressure $\geq 140 \mathrm{mmHg}$ (BENJAMIN et al., 2018). Systemic hypertension is usually associated with other metabolic disorders, such as obesity, diabetes, and dyslipidemia, which increase the risk of cardiovascular diseases (Neves et al., 2013). Dietary intervention as a complementary approach to drug therapy, for people with both drug-treated and -untreated hypertension, could be useful to lessen metabolic disorders, and therefore to limit the risk of cardiovascular disease onset.

To date, the literature about the effect of food rich in ALA on blood pressure and associated metabolic disorders is controversial and hardly comparable due to variability of food matrix used, administered quantities, and enrolled patient types. For instance, a recent paper of PIETERS and co-workers (2019) stated that high intake of ALA (10 g of flaxseed oil daily, providing 4.7 g ALA) for 12 weeks, does not significantly affect blood pressure in subjects with pre-hypertension. On the contrary, a previous paper of TOSCANO and co-workers (2014) demonstrated that $35 \mathrm{~g}$ /day of chia flour (which provides approximately $20 \mathrm{~g}$ of ALA)

\footnotetext{
* To whom correspondence should be addressed.

Phone: +390761 357672; e-mail: lara.cost@libero.it
} 
for 12 weeks reduces blood pressure, lipid peroxidation, and plasma nitrite levels in both treated and untreated hypertensive individuals. However, similar changes in blood pressure and metabolic markers have not been found in overweight or obese adults who dailyconsumed 50 and $25 \mathrm{~g}$ of chia seeds for 12 weeks and 10 weeks, respectively (NIEMAN et al., 2009; 2012).

Chia seeds (Salvia hispanica L.), considered as oilseeds due to their high oil content (25-40\% of total fats), are among the plant sources with the highest content of ALA, up to $68 \%$, compared to the most widely used flaxseed (57\%) (AYERZA \& COATES, 2011). Moreover, chia seeds contain other nutritionally relevant compounds, such as antioxidants (chlorogenic acid, caffeic acid, myricetin, quercetin, and kaempferol), gluten free protein containing all the essential amino acids, dietary fibres, and minerals (COSTANTINI et al., 2014).

Despite the fact that human studies on the effects of chia seeds in treated and untreated pathological people have been done, study concerning chia seed effects on subjects with the overt pathology of hypertension and without drug interference cannot be performed for ethical reasons. Therefore, considering the conflicting results in the literature on human volunteers, and in order to begin to shed light on the mechanism by which ALA-rich chia seeds can or cannot counteract the cardiometabolic risk factors, the aim of the present paper has been to study, for the first time, the plasma biochemical markers of cardiometabolic diseases in the animal model Spontaneously Hypertensive Rats (SHRs) with the overt pathology of hypertension, through a five-week $5 \%$ chia seed supplementation and without drug interference. Moreover, a comparison to common raw materials, wheat and corn, poor of fatty acids and containing linoleic acid (LA, C18:2 n-6), respectively, were done. The endogenous vasoconstrictor ET-1 and the endogenous vasodilators $\mathrm{NO}$ and BK, that are cardiometabolic markers normally altered in pathological subjects, were analysed. Furthermore, considering that the omega-3 ALA is highly prone to oxidation (DELANY et al., 2000), plasma antioxidant capacity, by hydroxyl radical antioxidant capacity (HORAC) and ferric reducing antioxidant power (FRAP) methods, and inflammatory condition through the CRP in the serum were analysed.

\section{Materials and methods}

\subsection{Materials}

SHRs were purchased from Charles River Laboratory, Lecco, Italy. Casein, salt mixture, vitamin mixture, choline, and methionine were purchased from Laboratories Dottori Piccioni, Milano. Tubes containing ethylene-diamine-tetra-acetic acid (EDTA), sodium citrate, or heparin were purchased from Venosafe Terumo, Leuven Belgium. The ET-1 and BK levels were analysed by immunoassay kits (ENZO life Sciences). Corning Spin-X concentrators were purchased from Corning Incorporated, MA, USA. Plasma NO metabolite levels $\left(\mathrm{NO}_{2}\right.$ and $\mathrm{NO}_{3}$ ) were analysed by colorimetric assay kit (ENZO life Sciences). HORAC assays were manufactured by Oxford Biomedical Research (Oxford, MI, USA). The CRP levels were analysed by immunoassay kits (Alpha diagnostic international). Chia seeds (Salvia hispanica L.) were obtained from Alesco S.r.l. (Pisa, Italy). Wheat and corn seeds were purchased from a local market. All other chemical reagents were purchased from SigmaAldrich (St. Louis, USA). 


\subsection{Experimental animal and diets}

Fifteen twenty-five-week-old male SHRs were used. In the experimental period, the rats were housed in individual cages ( $1 \mathrm{rat} / \mathrm{cage}$ ) under conventional conditions of adequate temperature $\left(23 \pm 2{ }^{\circ} \mathrm{C}\right)$ and humidity $(60 \%)$ with a 12 -h light/dark cycle with free access to food and tap water. After an acclimatization cycle of 7 days, an experimental period of five weeks was started, and SHRs were randomly divided into three groups $(n=5)$, namely "wheat" rats, "corn" rats, and "chia" rats, and every day they were ad-libitum fed with a diet formulated in accordance with AIN-93 (48\% wheat flour, 17\% sucrose, 14\% casein, 10\% corn oil, 4\% salt mixture, $1 \%$ vitamin mixture, $0.6 \%$ choline, and $0.4 \%$ methionine). The diet was supplemented with $5 \%$ of grounded daily chia flour, corn flour, and wheat flour, respectively (approximately $1.56 \mathrm{~g}$ ALA and 0.51g LA for chia seed (CostANTINI et al., 2014) and $0.03 \mathrm{~g}$ LA for corn (INRAN, 2009)). Pellet intakes were recorded daily, while body weights were recorded weekly over the five-week experimental period. The treatment was stopped 1 day before the end of the experiment to study the long-term effects of the diet without acute administration effects. At the end of the experimental period, animals were fasted overnight, anesthetized with isofluorane vaporization (2-2.5\%), and euthanized by cardiac exsanguination and subsequent cervical dislocation. Blood was drawn from the heart in tubes containing EDTA, sodium citrate, or heparin. Plasma was separated by centrifugation at $3000 \times g$ for $20 \mathrm{~min}$ at $4{ }^{\circ} \mathrm{C}$, and the supernatant was utilized for analysis. All experiments and surgical procedures were performed in accordance with the ethical standards as formulated in the Helsinki Declaration of 1964 (revised in 2013) and the Italian Laws (D.L.vo 116/92 and following additions, which enforce EU86/609 directives (EEC, 1986), and with the approval of the Health Ministry (authorization number DGSAF 0009805-A-23/05/2012) and the animal care committee of Tuscia University.

\subsection{Plasma and serum biochemical analysis}

The antioxidant capacity was analysed using the HORAC and FRAP methods on plasma. A FRAP assay was performed according to the method described by BENZIE and STRAIN (1999), which was adapted for 96-well plates and an automatic reader (Infinite 2000, Tecan, Salzburg, Austria). Briefly, $160 \mu \mathrm{l}$ of FRAP assay solution (consisting of $20 \mathrm{mmol}$ ferric chloride solution, $10 \mathrm{mmol}$ of $\mathrm{Fe}^{3+}$ 2,4,6-tripyridyl-s-triazine - TPTZ - solution, and $0.3 \mathrm{M}$ acetate buffer at $\mathrm{pH}$ 3.6) was prepared daily, mixed with $10 \mu \mathrm{l}$ of the sample, standard, or blank, and dispensed into each well of a 96-well plate. The absorbance was measured at 595 $\mathrm{nm}$ at $37^{\circ} \mathrm{C}$ after $30 \mathrm{~min}$ of incubation. The final results are expressed as $\mu \mathrm{mol} \mathrm{Fe}{ }^{2+}$ equivalents per 1 of plasma, and the results were obtained using a standard curve with different concentrations of ferrous sulphate heptahydrate $\left(\mathrm{FeSO}_{4} \cdot 7 \mathrm{H}_{2} \mathrm{O}\right)$. The HORAC assay kit was used according to the manufacturer's instructions. The final results are expressed as mmol gallic acid equivalents (GAE) per 1 of plasma. The ET-1 and BK levels were analysed by immunoassay kits (endothelin-1 ELISA kit and bradykinin ELISA kit, respectively). The plasma $\mathrm{NO}$ metabolite $\left(\mathrm{NO}_{2}\right.$ and $\left.\mathrm{NO}_{3}\right)$ levels were analysed through a colorimetric assay kit. $\mathrm{NO}_{2}$ and $\mathrm{NO}_{3}$ are stable metabolites of $\mathrm{NO}$ after deproteinization of the plasma through concentrator-based filtration. The CRP levels were analysed in the serum by immunoassay kits. The concentration of CRP in samples and control is calculated from a curve of standards containing known concentrations of CRP. Results are expressed as $\mathrm{mmol}^{-1}$ plasma. 


\subsection{Statistical analysis}

Mean and standard deviation (SD) were calculated for all three rat groups $(n=5)$. All analyses were conducted in triplicate. Statistical analysis was performed with the XLSTAT 20134.03 (Addinsoft SARL, New York, USA) software using one-way ANOVA. Fisher's least significant difference test was used to describe statistical differences between means at significance level $\mathrm{P}<0.05$.

\section{Results and discussion}

In the present study, the effects of chia seeds on plasmatic antioxidant capacity and cardiometabolic parameters were evaluated after its five-week administration to SHR hypertensive animal model and compared with staple raw materials, wheat and corn. SHRs had the overt pathology of hypertension, their use allowed to study the effects of chia seeds on plasmatic cardiometabolic biochemical parameters without the interference of a drug treatment.

Pellet intakes were recorded daily, and no differences between groups were found (data not shown). The results shown in Figure 1 indicate that there were no weight differences between groups, even though the chia seed diet was higher in fats (total fats: $2.6 \mathrm{~g} / 100 \mathrm{~g}$ in wheat (INRAN, 2009), $3.8 \mathrm{~g} / 100 \mathrm{~g}$ in corn (INRAN, 2009), and $32 \mathrm{~g} / 100 \mathrm{~g}$ in chia seeds (Costantini et al., 2014)). Though differences in weights were not statistically significant, a trend, chia $<$ corn $<$ wheat, could be observed. This result is consistent with the results of CHICCO and co-workers (2009), who found that Wistar rats fed a 'sucrose rich diet' (SRD) supplemented with chia seeds showed a weight gain slightly lower than in the group of rats fed the SRD alone, even though a similar energy intake was recorded in both the SRD and SRD+chia seed groups. So, in relation to the present work, we could hypothesize that higher amounts of chia seed can lead to significant weight changes (CHICCO et al., 2009).

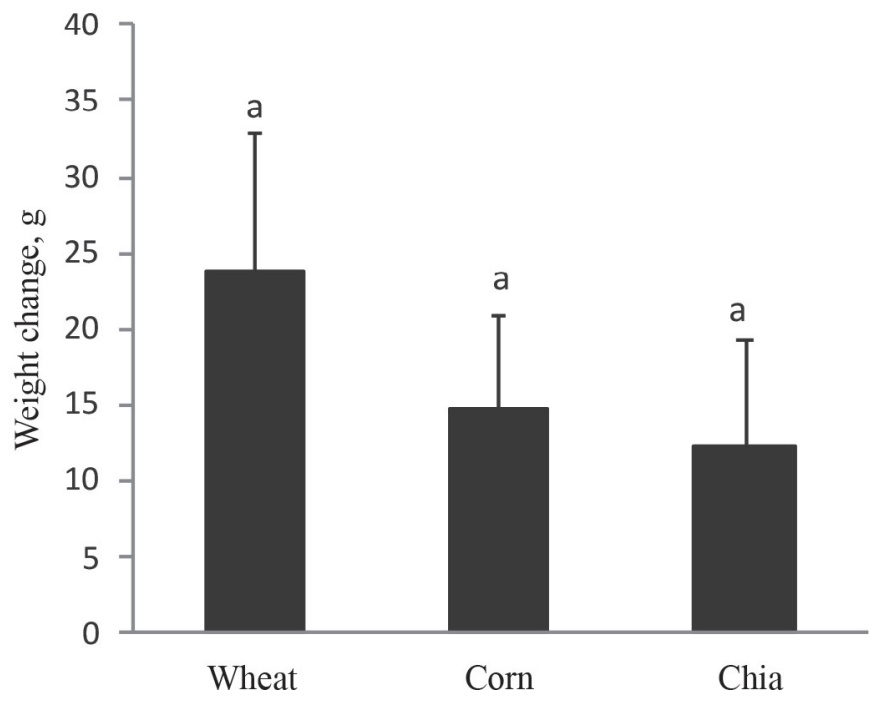

Fig. 1. Weight changes at the end of the experimental period. Each value is expressed as the mean \pm S.D. $(n=5)$. Different letters $(\mathrm{a}$ and $\mathrm{b})$ denote significant differences $(\mathrm{P}<0.05)$ within each panel. Wheat: rats fed with wheat supplement, corn: rats fed with corn supplement; chia: rats fed with chia supplement 
Both analyses of the total antioxidant capacity (FRAP Fig. 2A and ORAC Fig. 2B) showed that the experimental chia group was statistically $(\mathrm{P}<0.05)$ more oxidized than the control wheat group $\left(712 \pm 98 \mu \mathrm{mol} \mathrm{Fe}{ }^{2+} / 1\right.$ and $34 \pm 1 \mathrm{mmol} \mathrm{GAE} / 1$ respectively, for chia group; $877 \pm 79 \mu \mathrm{mol} \mathrm{Fe}{ }^{2+} / 1$ and $41 \pm 6 \mathrm{mmol} \mathrm{GAE} / 1$, respectively, for wheat group). However, there was no difference $(\mathrm{P}<0.05)$ between chia group and corn group for either total antioxidant capacity analysis $\left(814 \pm 103 \mu \mathrm{mol} \mathrm{Fe}{ }^{2+} / 1\right.$ and $37 \pm 5 \mathrm{mmol}$ GAE/l respectively, for corn group). This may be due to the higher intake of omega- 3 fatty acids that determined the spread of radical species already present in these pathological subjects. These results are in contrast with the results of MARINELI and co-workers (2015), who found a reduced oxidative stress in Wistar diet-induced obese rats (high fat and high fructose diet) after 6 and 12 weeks of 10\% chia seed supplementation. However, it should be noted that the oxidative stress in animal models with a chronic pathological condition is consolidated in comparison with animal models with induced pathological condition.
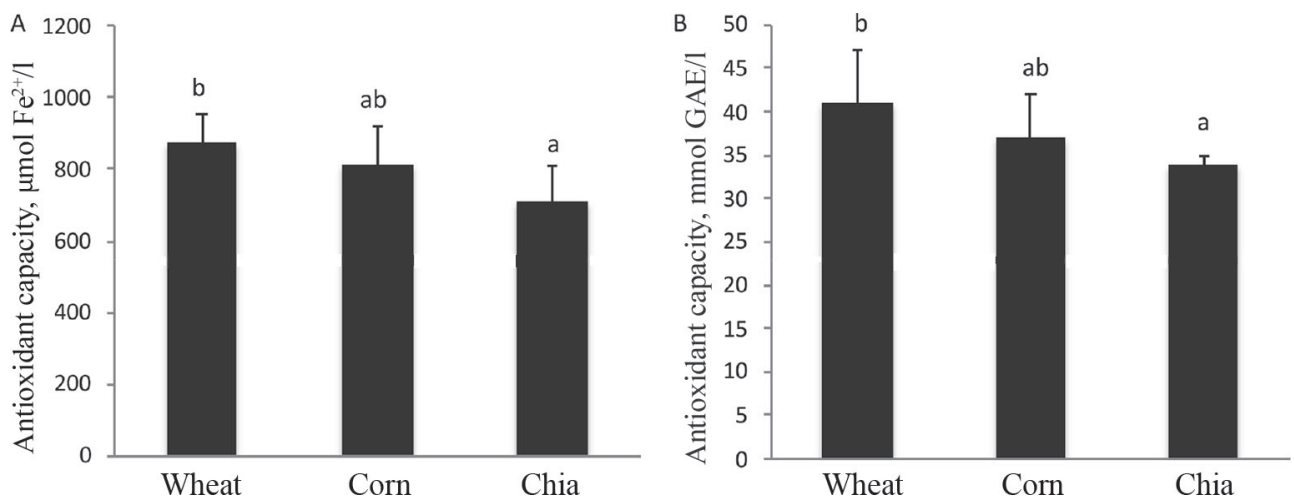

Fig. 2. The plasma total antioxidant capacity determined by FRAP (panel A) and ORAC (panel B). Each value is expressed as the mean \pm S.D. $(n=5)$. Different letters ( $a$ and $b)$ denote significant differences $(P<0.05)$ within each panel. Wheat, corn, chia: see Figure 1.

Anyway, even if the chia group was more oxidized than the control wheat group, this condition apparently does not trigger an inflammatory response; indeed, there was no difference between groups for serum CRP (Fig. 3A). Similarly, TosCANO and co-workers (2014) found no differences for the inflammatory marker high-sensitivity CRP between experimental groups of chia supplementation and placebo. On the contrary, POUDYAL and coworkers (2012) demonstrated that Wistar rats fed high carbohydrate and high fat diet possess a CRP amount almost doubled compared to the rats that have followed the same diet, but supplemented with $5 \%$ of chia seeds. Further studies are needed to understand the real action of chia seeds in relation to inflammatory condition. However, these preliminary data may reflect an anti-inflammatory action related to the high ALA amount found in chia seeds (Poudyal et al., 2012).

Finally, in the present study, for the first time, the plasma functional activity of chia seeds was compared analysing some cardiometabolic mediators normally altered in pathological individuals and in pathological animal model, SHRs. The found results showed that among the analysed biochemical parameters, the endogenous vasoconstrictor ET-1 significantly decreased $\left(29 \pm 2 \mathrm{pg} \mathrm{ml}^{-1}\right.$ for chia seed group against $37 \pm 5$ and $42 \pm 2 \mathrm{pg} \mathrm{ml}^{-1}$ for corn and wheat groups, respectively; Fig. 3B). For the vasodilators NO and BK (Fig. $3 \mathrm{C}$ and 
D, respectively), no significant differences were observed, even if there was a trend of increase for NO, but it was not statically significant $\left(39 \pm 11 \mu \mathrm{mol}^{-1}\right.$ for chia seed group against $32 \pm 6$ and $31 \pm 16 \mu \mathrm{mol} 1^{-1}$ for corn and wheat groups, respectively; Fig. 3B). Four previous studies have evaluated the effects of chia seeds in relation to cardiometabolic diseases, but not considering BK and ET-1. Among these, two studies carried out on overweight adults found no changes in CRP, plasma cytokines, blood lipoproteins, serum cholesterol, and blood pressure after a chia seeds supplementation of $50 \mathrm{~g} \mathrm{day}^{-1}$ and $25 \mathrm{~g}$ day $^{-1}$ for 12 and 10 weeks, respectively (NIEMAN et al., 2009; 2012). The other two papers similarly found a mean systolic blood pressure decrease of $6.3 \mathrm{mmHg}$ in diabetic patients and up to $11.2 \mathrm{mmHg}$ in drug-treated hypertensive patients after a $37 \mathrm{~g} \mathrm{day}^{-1}$ and $35 \mathrm{~g}^{\text {day }^{-1}}$ supplementation, respectively, for 12 weeks (VuKSAN et al., 2007; TOSCANO et al., 2014). Moreover, these two papers found a reduction in the plasma nitrite and malondialdehyde levels, but not changes for CRP in drug-treated hypertensive patients (TosCANO et al., 2014), and a reduction in CRP and vonWillebrand factor levels in diabetic patients (VUKSAN et al., 2007).
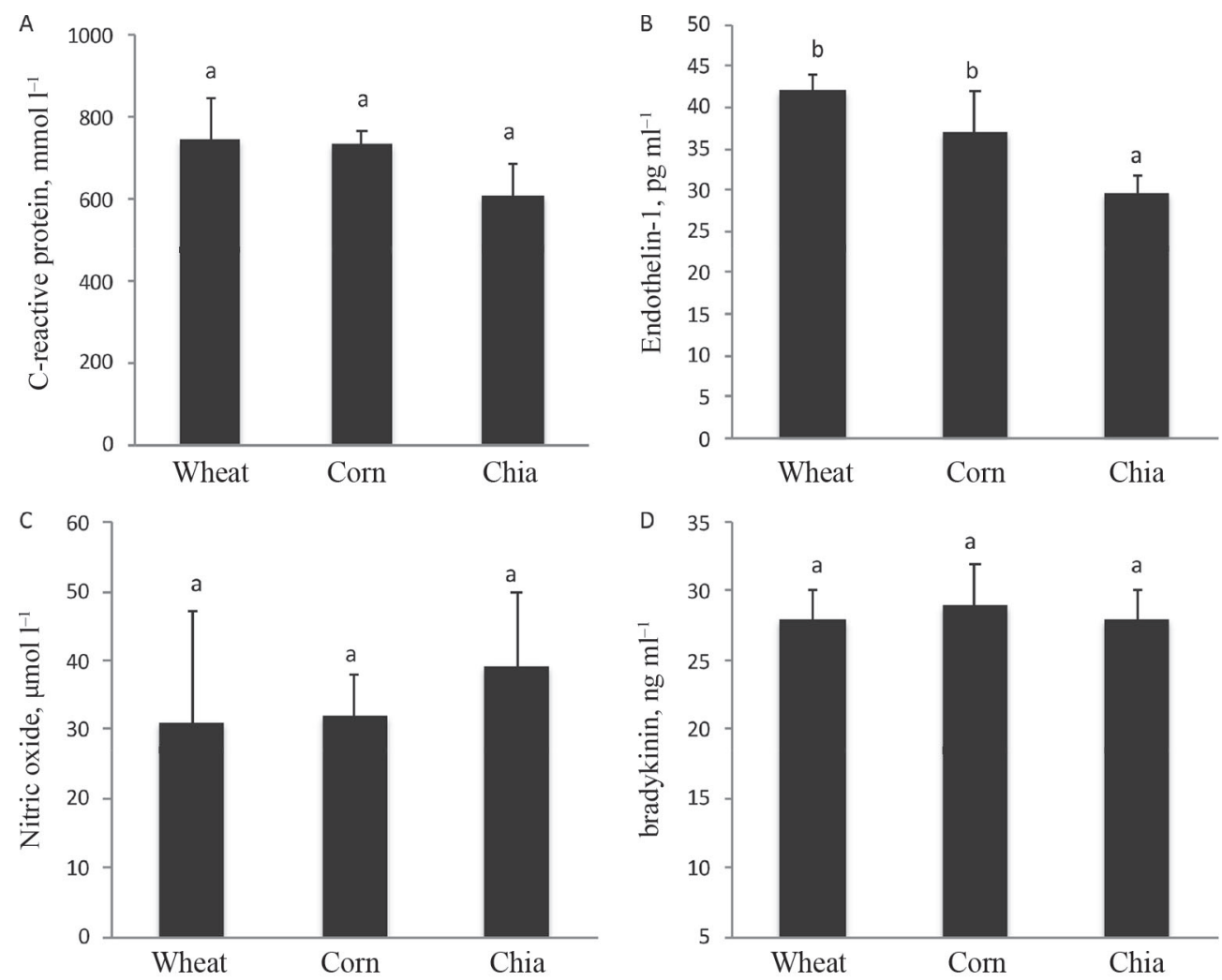

Fig. 3. Plasma inflammation parameter CRP (A) and plasma blood pressure parameters (B: endothelin-1; C: nitric oxide; D: bradykinin). Wheat, corn, chia: see Figure 1. Each value is expressed as the mean \pm S.D. ( $n=5)$. Different letters $(\mathrm{a}$ and $\mathrm{b})$ denote significant differences $(\mathrm{P}<0.05)$ within each panel. 


\section{Conclusions}

In the present study, a potential health effect of chia seeds in animal model with an overt pathology of hypertension and without drug interference has been highlighted, providing a significant decrease of the endogenous vasoconstrictor ET-1, even though oxidation occurred as side effect. This study suggests that higher quantities of chia seeds may have a higher impact on biochemical markers of cardiometabolic diseases. At the same time, the combination of chia seeds with another raw material having high antioxidant capacity could prevent the oxidation of the high amount of polyunsaturated fatty acids of chia seeds (CosTANTINI et al., 2014), highly unstable and susceptible to the high oxidation already present in this pathologic condition.

This study was financially supported in part by a grant from the Agriculture Ministry (MIPAF) ALIMED Project $(\operatorname{cod} 7110 / 730303 / 10)$.

The authors declare no competing financial interest.

\section{References}

Ayerza, R. \& CoAtes, W. (2011): Protein content, oil content and fatty acid profiles as potential criteria to determine the origin of commercially grown chia (Salvia hispanica L.). Ind. Crop. Prod., 34, 1366-1371.

Benjamin, E.J., Virani, S.S., Callaway, C.W., Chamberlain, A.M., Chang, A.R., .. \& Muntner, P. (2018): Heart disease and stroke statistics - 2018 update: A report from the American Heart Association. Circulation, 137, E67-E492.

BenzIE, I.F.F. \& Strain, J.J. (1999): Ferric reducing/antioxidant power assay: Direct measure of total antioxidant activity of biological fluids and modified version for simultaneous measurement of total antioxidant power and ascorbic acid concentration. Methods Enzymol., 299, 15-27.

Chicco, A.G., D’Alessandro, M.E., Hein, G.J., Oliva, M.E. \& Lombardo, Y.B. (2009): Dietary chia seed (Salvia hispanica L.) rich in $\alpha$-linolenic acid improves adiposity and normalises hypertriacylglycerolaemia and insulin resistance in dyslipaemic rats. Br. J. Nutr., 101, 41-50.

Costantini, L., Lukšič, L., Molinari, R., Kreft, I., Bonafaccia, G., Manzi, L. \& Merendino, N. (2014): Development of gluten-free bread using tartary buckwheat and chia flour rich in flavonoids and omega-3 fatty acids as ingredients. Food Chem., 165, 232-240.

DeLany, J.P., Windhauser, M.M., Champagne, C.M. \& Bray, G.A. (2000): Differential oxidation of individual dietary fatty acids in human. Am. J. Clin. Nutr., 72, 905-911.

EEC (1986): Council Directive 86/609/EEC of 24 November 1986 on the approximation of law, regulations and administrative provisions of the member states regarding the protection of animals used for experimental and other scientific purposes, OJ. No. L., 358/1

INRAN (2009): National Research Institute for Food and Nutrition) 'Food composition tables'. Available at http:// nut.entecra.it/646/tabelle_di_composizione_degli_alimenti.html. (last accessed 3 April 2019).

Marineli, R. DA S., Lenquiste, S.A.A., Moraes, É.A. \& MArósticA, M.R. (2015): Antioxidant potential of dietary chia seed and oil (Salvia hispanica L.) in diet-induced obese rats. Food Res. Int., 76, 666-674.

Neves, M.F., Virdis, A., SAnjuliani, A.F. \& TibiriçÁ, E.V. (2013): Hypertension and cardiometabolic risk factors. Int. J. Hypertens., 2013, 634798.

Nieman, D.C., Cayea, E.J., Austin, M.D., Henson, D.A., McAnulty, S.R. \& Jin, F. (2009): Chia seed does not promote weight loss or alter disease risk factors in overweight adults. Nutr. Res., 29, 414-418.

Nieman, D.C., Gillitt, N., Jin, F., Henson, D.A., Kennerly, K., ...\& Schwartz, S. (2012): Chia seed supplementation and disease risk factors in overweight women: A metabolomics investigation. J. Altern. Complem. Med., 18, 700-708.

Pieters, D.J., Zock, P.L., Fuchs, D. \& Mensink, R.P. (2019): Effect of $\alpha$-linolenic acid on 24-h ambulatory blood pressure in untreated high-normal and stage I hypertensive subjects. Br. J. Nutr., 121, 155-163. 
Poudyal, H., Panchal, S.K., WaAnders, J., Ward, L. \& Brown, L. (2012): Lipid redistribution by $\alpha$-linolenic acidrich chia seed inhibits stearoyl-CoA desaturase-1 and induces cardiac and hepatic protection in diet-induced obese rats. J. Nutr. Biochem., 23, 153-162.

Toscano, L.T., da Silva, C.S.O., Toscano, L.T., DE Almeida, A.E.M., Da Cruz Santos, A \& Silva, A.S. (2014): Chia flour supplementation reduces blood pressure in hypertensive subjects. Plant Food. Hum. Nutr., 69, 392-398.

Vuksan, V., Whitham, D, Sievenpiper, J.L., Jenkins, A.L., Rogovik, A.L., ...\& Hanna, A. (2007): Supplementation of conventional therapy with the novel grain Salba (Salvia hispanica L.) improves major and emerging cardiovascular risk factors in type 2 diabetes: Results of a randomized controlled trial. Diabetes Care, 30, 2804-2810.

Open Acces statement. This is an open-access article distributed under the terms of the Creative Commons Attribution-NonCommercial 4.0 International License (https://creativecommons.org/licenses/by-nc/4.0/), which permits unrestricted use, distribution, and reproduction in any medium for non-commercial purposes, provided the original author and source are credited, a link to the CC License is provided, and changes - if any - are indicated. 